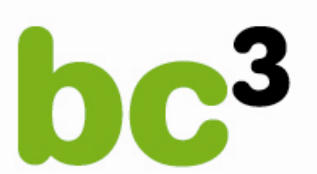

BASQUE CENTRE

FOR CLIMATE CHANGE

Klima Aldaketa Ikergai

\title{
Endogenous Timing in Pollution Control: \\ Stackelberg versus Cournot-Nash Equilibria
}

\author{
Melanie Heugues
}

February 2011

BC3 WORKING PAPER SERIES

2011-03 
The Basque Centre for Climate Change (BC3) is a Research Centre based in the Basque Country, which aims at contributing to long-term research on the causes and consequences of Climate Change in order to foster the creation of knowledge in this multidisciplinary science.

The $\mathrm{BC} 3$ promotes a highly-qualified team of researchers with the primary objective of achieving excellence in research, training and dissemination. The Scientific Plan of BC3 is led by the Scientific Director, Prof. Anil Markandya.

The core research avenues are:

- Adaptation to and the impacts of climate change

- Measures to mitigate the amount of climate change experienced

- International Dimensions of Climate Policy

- Developing and supporting research that informs climate policy in the Basque Country

See www.bc3research.org for further details.

The BC3 Working Paper Series is available on the internet at http://www.bc3research.org/lits_publications.html

Enquiries (Regarding the BC3 Working Paper Series):

Roger Fouquet

Email: roger.fouquet@bc3research.org

www.bc3research.org

The opinions expressed in this working paper do not necessarily reflect the position of Basque Centre for Climate Change (BC3) as a whole.

Note: If printed, please remember to print on both sides. Also, perhaps try two pages on one side. 


\title{
Endogenous Timing in Pollution Control: Stackelberg versus Cournot-Nash Equilibria
}

\author{
Melanie Heugues ${ }^{1}$
}

In the framework of international cooperation on climate change to control greenhouse gas emissions $(G H G)$, this paper aims to shed new light on the eventuality of the emergence of a country (or a group of countries) behaving as a leader in the implementation of its environmental policy. The sequence of moves in the existing literature is usually an exogenous assumption, - known as the Cournot assumption (if countries take action simultaneously) and the Stackelberg assumption (if they act sequentially, the latter observing the strategy of the former). The main purpose here is to make the timing endogenous. To do so, we introduce a pre-play stage in the basic two-country game. Then we provide different sets of minimal conditions - on the benefit and damage functions linked to GHG emissions into the atmosphere, yielding respectively the simultaneous and the two sequential modes of play. While the results essentially confirm the prevalence of the former, they also indicate that the latter are natural under some robust conditions: a leader can emerge endogenously when implementing its environmental policy. Finally we provide sufficient conditions for a specific leader to appear. All the results come with an analysis in terms of global emissions and global welfare. No extraneous assumptions such as concavity, existence, or uniqueness of equilibria are needed, and the analysis makes crucial use of the basic results from the theory of supermodular games.

Keywords: Climate change; non cooperative game; global pollution; strategic interactions; endogenous timing; supermodular game theory.

JEL Classification: C72, H41, Q54

Cite as: Heugues, M. (2011) Endogenous Timing in Pollution Control: Stackelberg versus CournotNash equilibria, BC3 Working Paper Series 2011-03. Basque Centre for Climate Change (BC3). Bilbao, Spain.

\footnotetext{
${ }^{1}$ Basque Centre for Climate Change (BC3). Alameda Urquijo, 4 - 4, 48008 Bilbao, Spain. Corresponding author E-mail:melanie.heugues@bc3research.org.
} 


\section{Introduction}

Since the "Earth Summit" of Rio de Janeiro in 1992, almost all countries of the world are part of the United Nations Framework Convention on Climate Change (UNFCCC). ${ }^{2}$ This Convention recognizes the existence of climate change and sets general goals and rules for stabilizing greenhouse gas (GHG) concentrations. Among its general principles, members of the Convention have agreed to place the heaviest burden for fighting climate change on industrialized nations - since they are the source of most past and current GHG emissions -, and to develop national programs to slow climate change in sectors such as agriculture, energy, transportation and so on. They also recognized that the Convention was a "framework" document that had to be amended or augmented over time so that efforts to deal with global warming and climate change can be focused and made more effective. ${ }^{3,4}$

Nevertheless, the current negotiation process is rather long and complex with a permanent risk of failure. The distinctive characteristics of the problem turn it into a big challenge. First of all, the environment - or the atmosphere, is a global public good that countries are free to provide or to enjoy freely. ${ }^{5}$ Secondly, States are sovereign and no supranational authority exists to implement a globally optimal environmental policy: each country has thus to decide voluntarily to reduce its GHG emissions given a strong incentive to free ride. Finally, even if countries agree on the existence of the problem and its urgency, national emissions are a strategic variable since they are linked to national economic activities, and thus to economic growth and development. ${ }^{6}$

Actually, two arguments exist for and against a strategy to implement stronger or more lax national environmental policies than the others (Ulph, 2001). Because countries are linked through trade, environmental policies will affect the international competitiveness of particular sectors of an economy. If governments are concerned about this loss of competitiveness, they may set too lax environmental policies, essentially as a form of covert protection. An alternative point of view is that such strategic trade considerations may also induce governments to set tough environmental policies to give their domestic producers a competitive advantage in developing "green" technologies ahead of their rivals. In all, an important consideration for a country will be the economic cost of having to set tougher environmental policies than at least some other countries.

Enhancing worldwide economic progress combined with reduced emissions is a rather hard task when each country is pursuing its own interest. In this framework, we aim to study the eventuality of the emergence of a country or a group of countries acting as a leader in the implementation of its

\footnotetext{
${ }^{2}$ The UNFCCC is an international treaty joined by 192 countries around the world. Countries ratifying the treaty are called "Parties to the Convention". The Conference of the Parties (COP), as an association of all member countries, is the prime authority of the Convention. To know more, see the website http://unfccc.int/2860.php.

${ }^{3}$ To tackle the problem of GHG accumulation in the atmosphere, governments of the world meet annually for a period of two weeks. They evaluate the status of climate change and the effectiveness of the treaty.

${ }^{4}$ In 1997, the Convention led in turn to the adoption of the Kyoto Protocol.

${ }^{5}$ The global character means that there is not a unique well identified and settled agent responsible for GHG emissions. Emissions are rather the indirect consequence of the performance of a large group of economies.

${ }^{6}$ Negotiations are also the place to defend national economic interests (given the increasing inter-connexion of countries on global markets) and geostrategic positions.
} 
environmental policy. To illustrate our purpose, we note that the last Conference of the Parties (COP15, Copenhagen, 2010) has been the occasion of intense discussions on United States and China's respective GHG emissions: in which respect could we observe the emergence of leading behaviour from one of the two largest emitters of the planet? Conversely, the European Union was among the most involved in the process to promote cooperation by offering to strongly curb its GHG emissions and also trying to initiate an agreement among a more limited number of countries for the latter attempt to be more effective. ${ }^{7}$

How can such behaviour be explained? Would it be a sacrifice of the leading country or group to implement such a policy? Is there no economic rationale? What would the consequences be at a global level (in terms of effectiveness) and what would the strategy adopted by the others be in response? The aim of this paper is to shed light on the economic circumstances justifying such behaviour, i.e. leading in the implementation of one's national environmental policy. The results exposed are built on the literature studying international cooperation on global issues using non cooperative game theory. ${ }^{8}$ This tool has shown to be particularly suited to model strategic interactions among States. The fundamental model is the one introduced by Carraro and Siniscalco (1993) and called "the global emission game" (Finus, 2001). ${ }^{9}$ The game depicts the problem of GHG accumulation in the atmosphere and linked to human activities. Broadly speaking, emissions are viewed as an output of the production and consumption of goods from which countries derived benefits, while payoff functions describe the net welfare implications from emissions.

As the game involves only two countries (or two groups), two alternatives exist when considering the sequence of moves in the global emission game: a simultaneous and a sequential move version of the constituent game. In the former case, countries choose their emission level at the same time whereas in the latter, one country chooses first, the second mover observing the decision of the former. In the first game, equilibria are called "Cournot-Nash equilibria" while in the second one, they are named "Stackelberg equilibria". Both have been postulated and studied independently in the literature. Comparing outcomes of each game yields some differences (Finus, 2001). ${ }^{10}$

Nonetheless, the main drawback of the sequential game is that the order of move is generally exogenously attributed to the countries, while the latter are a priori interchangeable. Hence, a general

\footnotetext{
${ }^{7}$ The question on the prospects of seeing a leader emerging in the implementation of its environmental policy can also be relevant with respect to other groups: Annex I and non-Annex I countries; signatories and non signatories of a potential Post-2012 Kyoto Protocol; or even among OECD members (inside Annex I countries).

${ }^{8}$ Note that cooperation can even emerge in a non cooperative framework (Carraro and Siniscalco, 1993; Barrett, 1994; Diamantoudi and Sartzetakis, 2006 and Heugues, 2009a).

${ }^{9}$ An alternative game is the one in abatement proposed by Hoel (1991) or Barrett (1994). Both games are strictly equivalent as soon as countries' individual emissions are always positive (Diamantoudi and Sartzetakis, 2006).

${ }^{10}$ When countries choose their emission level non cooperatively, the country which takes action first, i.e. the leader, has an advantage on the second mover, i.e. the follower, with regard to the case where both countries take action simultaneously: he can implement its best outcome. Hence, from a single country's perspective the sequential move is clearly preferable, provided he can move first.
} 
problem of the Stackelberg equilibrium concept is the motivation of the assumption that one agent (or group of agents) moves first, that is, has superior information compared to the follower(s). Another point raised by Finus (2001) is that, since each country has an incentive to be the leader, one would expect all countries to compete for this position. Therefore, as long as this leader-follower relationship is not obvious from the investigated problem itself, some doubts about the reason for such an asymmetry remain. If each country assumes the supposed leadership and chooses the corresponding emission level, both countries may end up in a situation which cannot be an equilibrium point. Hence, it seems plausible to expect that in this case countries are led back to the Cournot-Nash equilibrium.

One purpose of this paper is to show that the reasoning above is incomplete. Specifically, we have to relate the payoff of the simultaneous game with the one of the follower in the sequential move game. If there are conditions under which the latter dominates the former, then the sequential global emission game becomes the appropriate representation of the game.

More generally, this paper provides different sets of minimal conditions on the countries' payoff functions yielding respectively the simultaneous and the two sequential modes of play. To do so we construct an extended game of the global emission game: we introduce a previous stage to the global emission game in which both countries decide simultaneously on the date ("Early" or "Late") at which they want to play the constituent game. ${ }^{11}$ If both countries choose the same date, they'll play simultaneously; if the dates are distinct, they'll play sequentially with the order announced. Note that, in the second stage, countries choose their own emission level and fulfil any commitments made in the first stage. We thus assume that countries can commit. ${ }^{12}$ While our findings essentially confirm the predominance of the simultaneous game, they also indicate that the sequential game under perfect information is natural under some robust conditions. The key determinant of the analysis is the nature of the interactions between countries. ${ }^{13}$

Finally, the results established in the paper rely on the theory of supermodular games. By this approach, no assumptions such as concavity of payoffs, existence or uniqueness of equilibria are needed and it lets us extend the usual framework of the literature. ${ }^{14,15}$

The remainder of the paper is organised as follows. Section 2 describes the extended global emission game. Section 3 defines the solution concepts as well as the conditions of their existence. In section 4 , the main results together with their interpretation are presented. Section 5 concludes.

\footnotetext{
${ }^{11}$ This procedure is drawn from the literature on industrial organization. See Hamilton and Slutsky (1990) or Amir and Grilo (1999) in the case of a duopoly game.

${ }^{12}$ Other assumptions on countries' capability to commit would lead to other results with certainty (Boadway et al., 2007). An extension would be to consider the consequences of only one country being able to commit.

${ }^{13}$ The global emission game can be seen as a reduced form of the framework in Copeland and Taylor (2005) justifying to consider both kinds of interactions: substitutable and complementary. This point is also developed in Barrett $(2003,2005)$.

${ }^{14}$ The approach can be applied in both cases of strategic complementarity and substitutability as the game considered is a two-player game.

${ }^{15}$ In more to Appendix A, the interested reader can refer to Amir (2005), Topkis (1998), Vives (1999) or Cooper (1999) to have a larger view on the interest of this mathematical tool in economics.
} 
Appendix A contains a summary of the lattice-theoretic notions we rely on, whereas Appendix B provides all the proofs and some intermediate results.

\section{The extended global emission game}

The game we consider in this section aims at determining the conditions under which a leader can emerge endogenously in the implementation of its national environmental policy. Until now to the best of our knowledge, this kind of behaviour has always been postulated exogenously in the referred literature. ${ }^{16}$ Instead, we introduce the possibility for countries to choose their position in the constituent game - named "the global emission game" (Finus, 2001). The game as a whole - hereafter named "the extended global emission game" - is a two-country, two-stage game. ${ }^{17}$ The timing is such that in the first stage, both countries decide simultaneously and independently the date they want to play in the second stage: "Early" or "Late". In the second stage, countries play the global emission game, i.e. choose their individual emission level, maximising a payoff function defined below. ${ }^{18}$

Formally, consider two countries not necessarily identical such that $i=1,2$ and $N=2$. Linked to economic activities, each of them emits GHGs that mix uniformly in the atmosphere. We note $x$ and $y$ respectively as the emission levels of Country 1 and of Country 2. $X_{i}=\left[0, K_{i}\right]$ stands for the strategy set of country $i$ and is a compact interval of the reals. $K_{i}$ accounts for a capacity constraint in terms of pollution. It means that country $i$ cannot infinitely produce GHG emissions or that its economic activities are bounded. In its most general form, the payoff function of country $i, f_{i}, i=1,2$, is expressed as the difference between the benefits of its own emissions, $B_{i}(\cdot)$, and the damages linked to global emissions, $D_{i}(\cdot) .{ }^{19}$ Then countries 1 and 2 respectively have payoffs:

$$
f_{1}(x, y)=B_{1}(x)-D_{1}(x+y) \quad \text { and } \quad f_{2}(x, y)=B_{2}(y)-D_{2}(x+y) .
$$

To establish our results, we assume throughout that $B_{i}(\cdot)$ and $D_{i}(\cdot)$ are twice continuously differentiable and non decreasing, $i=1,2$. The assumption of differentiable payoff functions is only made to simplify the analysis. Given the choice made in the first stage of the extended game by both countries, the second stage game is played simultaneously or sequentially:

i) If both countries choose the same date, the global emission game is then played simultaneously: each country determines its emission level given the emission level of the other. The resulting pure strategies Nash equilibria are named "Cournot-Nash equilibria";

\footnotetext{
${ }^{16}$ Seminal works assume for example that a country has a greater environmental consciousness than the other (Finus, 2001; Pereau et al., 2002); or in the literature on international environmental cooperation, that the coalition of signatories holds more information than the non signatories (Barrett, 1994; Diamantoudi and Sartzetakis, 2006).

${ }^{17}$ We call it "the extended global emission game" as we introduce a pre-play stage to the global emission game.

${ }^{18}$ The sequence of moves supposes that both countries can commit to their announcement in the first stage.

${ }^{19}$ Emissions in a country generate an externality causing environmental damage in this country but also in the other. The damage function traduces both the public good character and the global character of the environment.
} 
ii) If the dates chosen differ, then the global emission game becomes a two-stage game of perfect information. The constituent game is played sequentially with the order of moves as announced: the country announcing to play "Early" chooses its emission level first, whereas the other (announcing "Late") chooses its emission level observing the decision of the former. In this case, the underlying equilibrium concept is the one of "Stackelberg equilibrium". ${ }^{20}$

Figure 1 below displays the extensive form of the associated game. Here $e$ and $l$ stand for "Early" and "Late" and 1 and 2 for countries' names.

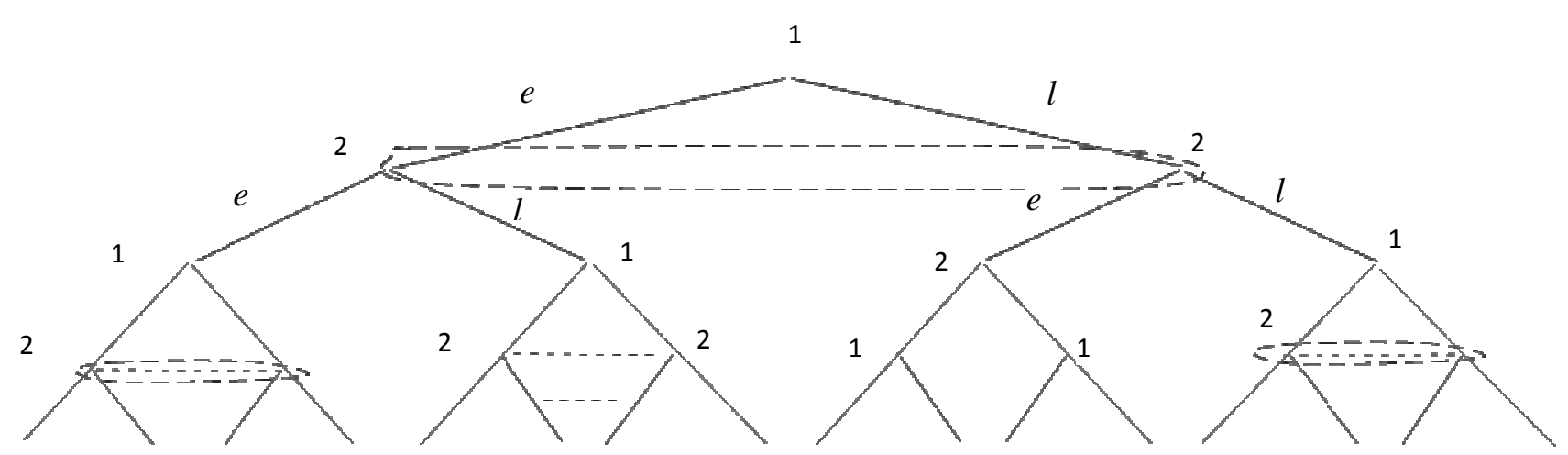

Figure 1: Extensive form of the extended global emission game. ${ }^{21}$

Figure 2 below displays the strategic form of the extended game. Notations are as follows: for $i=1$, 2 , let $f_{i}^{L}$ and $f_{i}^{F}$ respectively stand for the equilibrium payoffs of the leader and of the follower, whereas $f_{i}^{C N}$ stands for the equilibrium payoff of a country when both choose the same date in the preliminary stage.

\section{Country 2}

\begin{tabular}{lccc}
\cline { 2 - 3 } Country 1 & Early $(e)$ & Late $(l)$ \\
\cline { 2 - 3 } & Early $(e)$ & $f_{1}{ }^{C N}, f_{2}{ }^{C N}$ & $f_{1}{ }^{L}, f_{2}{ }^{F}$ \\
\cline { 2 - 3 } & Late $(l)$ & $f_{1}{ }^{F}, f_{2}{ }^{L}$ & $f_{1}{ }^{C N}, f_{2}{ }^{C N}$ \\
\hline
\end{tabular}

Figure 2: Strategic form of the extended global emission game.

\footnotetext{
${ }^{20}$ Strictly speaking, i) this case implies that the second stage of the extended game consists of two sub-stages; ii) the equilibrium concept of the sequential game is the one of subgame perfect equilibrium but the term of Stackelberg equilibrium is used to stay conform to the terminology of the literature.

${ }^{21}$ The dotted lines mean that strategy sets in the constituent game are compact intervals of $I R_{+}$
} 
Either if both countries choose to determine their emission level sooner or to postpone this decision, the equilibrium payoffs of the simultaneous game do not change: time does not matter when determining the payoffs. Note also that a country may not unilaterally choose to be a leader or a follower, though he may elect not to be the latter simply by deciding to move in the first period in the preliminary stage. Moreover, the situation in which both countries choose their first mover optimal action can never be an equilibrium outcome of the extended game.

Hence, instead of considering both simultaneous and sequential games independently, this extension of the global emission game allows capturing the notion of endogenous timing. The subgame perfect equilibria (SPE) of the extended game thus induce an endogenous sequencing of moves in the original game. Consideration is restricted to the SPE in pure strategies only. They arise from the comparison of equilibrium payoffs of the simultaneous game with those of the sequential game when a country is the leader and when he is the follower. All the cases that can arise are summed up in Proposition 0 below. The characterization of the SPE is very general in that the uniqueness of the Cournot-Nash and Stackelberg equilibria for the constituent game is not postulated. As we do not assume continuity of the best responses, the fact that each country prefers being a leader than a simultaneous player at equilibrium needs to be included as an assumption in our setting. ${ }^{22}$

For the given two-country constituent game, let $C^{N}$ denote the set of Cournot-Nash equilibrium strategies and $S_{i}$ denote the set of Stackelberg equilibrium strategies with country $i$ as the leader. Finally let $E$ denote the set of SPE of the extended game. With a slight abuse of notation, each element of $E$ will be written as a pair of timing announcements and a sequential or simultaneous play of the constituent game. For example $E=\left\{(e, e), C^{N}\right\}$ means that both countries choose to act early leading them to produce one of their Cournot-Nash equilibrium emission levels in the constituent game.

\footnotetext{
${ }^{22}$ This assumption is not restrictive as the global emission game belongs to the class of games for which this assertion is always satisfied (Amir and Grilo, 1999).
} 


\section{Proposition 0:}

Consider the two-country game with $C^{N} \neq \phi$ and $S_{i} \neq \phi, i=1,2$. When each country $i$ is better off at any point in $S_{i}$ than at any point in $C^{N}$, the set of pure strategy subgame perfect equilibria of the extended game is such that:

a) If country i's payoff is strictly higher at his least preferred point in $C^{\mathrm{N}}$ than at every point in $S_{j}, j \neq i$ for $j, i=1,2$, then $E=\left\{(e, e), C^{N}\right\}$.

b) If country i's payoff is strictly higher at any point in $S_{j}$ than at his most preferred point in $C^{N}$, $i=1,2$, then $E=\left\{(e, l), S_{1}\right\} \cup\left\{(l, e), S_{2}\right\}$.

c) If countries are such that, for example Country 1 is as in a) and Country 2 is as in b), then $E=\left\{(e, l), S_{1}\right\}$.

Proposition 0 confirms that even if both countries always prefer to be a leader, a solution of the simultaneous game does not necessarily emerge: we also need to compare the payoff of the follower with regard to the one of the simultaneous game. ${ }^{23}$ Given the three cases of Proposition 0 ( $a, b$ and $c$ ), we have to establish the conditions under which a country prefers its payoff as a follower to the one as a simultaneous player, i.e. $f^{F}<[>] f^{C N}$. To do so, the next section characterizes the set of CournotNash equilibria of the simultaneous constituent game and the set of Stackelberg equilibria of the sequential constituent game.

\section{Definition, existence and characterization of equilibria in the constituent game}

This section defines and characterizes the set of equilibria of the global emission game first when both countries choose their emission level simultaneously and when they choose it sequentially under perfect information. The characterization will rely on the sign of the cross partial derivative of payoff functions: if positive the objective reflects strategic complementarities and if negative it reflects strategic substitutes. In each case we show that at least one pure strategy Cournot-Nash equilibrium in the simultaneous game and at least one Stackelberg equilibrium in the sequential constituent game always exist.

\subsection{Definition of Cournot-Nash and Stackelberg equilibria}

Formally, in the simultaneous game, a Cournot-Nash equilibrium is a pair $\left(x^{N}, y^{N}\right)$ such that, for all $x, y \geq 0, f_{1}\left(x^{N}, y^{N}\right) \geq f_{1}\left(x, y^{N}\right)$ and $f_{2}\left(x^{N}, y^{N}\right) \geq f_{2}\left(x^{N}, y\right)$. In equilibrium no country has the incentive to deviate given the strategy of the other.

In the sequential game under perfect information, i.e. the follower observes the action of the leader before acting, a pure strategy for the leader (e.g. Country 1 ) is the choice $x \geq 0$, and a strategy for the

${ }^{23}$ Proposition 0 is only a translation of the Nash equilibria of the extended game. 
follower is the mapping $g:[0, K] \rightarrow[0, K]$. Any Stackelberg equilibrium is a subgame perfect Nash equilibrium of the two-stage game, i.e. a pair $\left(x^{S}, g^{S}(\cdot)\right)$ such that:

$$
\begin{array}{ll}
\text { i) } \quad f_{1}\left(x^{S}, g^{S}\left(x^{S}\right)\right) \geq f_{1}\left(x, g^{S}(x)\right) & \forall x \geq 0, \\
\text { ii) } f_{2}\left(x^{S}, g^{S}\left(x^{S}\right)\right) \geq f_{2}\left(x^{S}, y\right) & \forall y \geq 0 .
\end{array}
$$

Any Stackelberg equilibrium is such that $\left(x^{S}, y^{S}\right)$ lies on the follower's best response correspondence defined by $b r_{2}(x)=\operatorname{argmax}_{y \geq 0} f_{2}(x, y)$. Moreover there is no $x \geq 0, x \neq x^{s}$ such that $f_{1}(x, y)>f_{1}\left(x^{S}, y^{S}\right), \forall y \in b r_{2}(x)$. It means that there is no emission level other than $x^{S}$ leading to a higher payoff level for the leader. The leader when choosing its equilibrium emission level in the first stage takes into account the second stage best response of the follower $b r_{2}(x)$ in its objective function. The game is then solved by backward induction. Through this equilibrium strategy, the problem of maximization of the follower does not change with regard to the situation where both countries choose simultaneously.

In what follows we characterize the set of Cournot-Nash and Stackelberg equilibria of the global emission game given the assumptions on the benefit and damage functions. Only pure strategy equilibria are taken into account.

\subsection{Existence and characterisation of equilibria in each subgame}

We now define the conditions under which the sets of Cournot-Nash and Stackelberg equilibria are non empty. The analysis relies on the sign of the cross partial derivative of a country's payoff function in its strategy $x$ and in the strategy of the other $y$ : if negative, a country's strategies are substitutable; if positive, a country's strategies are complement. The former case means that a country does more as the other does less (negative feedback); the latter means that a country does more as the other does more (positive feedback) (Barrett, 2003). ${ }^{24}$ This sign is the key determinant of the overall analysis: given this sign, equilibrium behaviours and payoffs change. From now on, the analysis relies on two minimal and strictly distinct sets of assumptions on the benefit and damage functions. We expose them and then explain them (the details on the mathematical definition are exposed in Appendix B).

\section{Assumptions under $\mathrm{A}_{1}$ :}

- The damage function $D_{i}(\cdot),(i=1,2)$ is strictly convex, $\forall x, y \geq 0$ and

- $\exists \bar{Z}_{i} \in\left[0, K_{i}\right]$ such that $B_{i}(Z)-D_{i}(Z) \leq B_{i}\left(\overline{Z_{i}}\right)-D_{i}\left(\overline{Z_{i}}\right), \forall Z(i=1,2)$.

\footnotetext{
${ }^{24}$ The limit case is when the cross partial derivative is null: countries' strategies are independent, i.e. as a country does more/less, the other does nothing (no feedback).
} 


\section{Assumptions under $\mathbf{A}_{2}$ :}

- The damage function $D_{i}(\cdot),(i=1,2)$ is strictly concave, $\forall x, y \geq 0$ and $^{25}$

- The benefit function $B_{i}(\cdot),(i=1,2)$ is strictly concave, $\forall x, y \geq 0$.

Under the set denoted $A_{1}$, a country's strategies are strategic substitutes and best responses are strictly decreasing in the strategy of the other. In fact the strict convexity of the damage function (damages increase at an increasing rate) yields to payoff functions with strictly decreasing differences in $(x, y)$ whatever the assumption on the benefit function. The definition of $\bar{Z}_{i}$ lets us express the best response of country $i$ when the other pollute 0 .

Under the set $A_{2}$, a country's strategies are strategic complements and best responses are strictly increasing in the strategy of the other. The strict concavity of the damage function (damages increase at a decreasing rate) insures that payoff functions have strictly increasing differences in $(x, y)$. The strict concavity of the benefit function is needed to insure global consistency between individual and global emission levels (Heugues, 2009b). ${ }^{26}$

The next proposition presents the minimal conditions under which $C^{N}$ is non empty when both countries' strategies are substitutable. In this case, the use of lattice theory is appropriate only because the number of agents is limited to two. To use it, we consider for one country the opposite sign on its strategy set (Amir, 2005). For example if Country 2 chooses the emission level $-y$ instead of $y$, decreasing differences in $(x, y)$ become increasing differences in $(x,-y)$. This kind of argument cannot be generalized to games with more than two agents. ${ }^{27}$

\section{Proposition 1:}

Under the set of assumptions $A_{l}$, the two-country global emission game is a supermodular game with the order reversal on the strategy set of one country, and $C^{N}$ is non empty.

The strict convexity of the damage function alone yields to payoff functions with strictly decreasing differences in $(x, y)$. To establish the existence of an equilibrium, we can distinguish two sub-cases relying on the form of the benefit function. If the latter is concave, countries' strategies are weakly substitutable (the slope of the best responses is strictly less than one in absolute terms) and the equilibrium is unique. ${ }^{28}$ On the contrary, if the benefit function is convex, countries' strategies are strongly substitutable (best responses are strongly decreasing with a slope greater than one in absolute terms) and payoff functions are not anymore necessarily concave. In this case, the second assumption in the set $A_{1}$ ensures the existence of an asymmetric equilibrium in which one country has positive

\footnotetext{
${ }^{25}$ The empirical literature on the evaluation of the impacts of climate change has recognized the importance of non linearities in the climate change issue (Ortiz and Markandya, 2009). On way to do it is to consider a Sshaped damage function (Dumas and Ha-Duong, 2005).

${ }^{26}$ As $z=x+y$, if $x$ and $y$ are mutually increasing, $z$ must be increasing as well.

27 See Vives (1999) for an alternative argument leading to the same conclusions.

${ }^{28}$ The proof is immediate because of the concavity of the payoff function. This case is one of the most exploited in the literature quoted above because of this nice mathematical property.
} 
emissions, the rival producing no emission (Heugues, 2009b). The following corollary to Proposition 1 aims at characterizing the Cournot-Nash equilibria of the game under the set of assumptions $A_{1}$.

\section{Corollary 1:}

Under the set of assumptions $A_{l}$, the extreme selections of the best-response correspondence $b r_{i}(\cdot)$ are non increasing in the rival's emissions. Hence, $C^{N}$ includes the point $(\bar{x}, \underline{y})$ where Country 1 [Country 2] chooses its highest [lowest] emission level in $C^{N}$. Furthermore, this point lies on $\underline{b r}_{2}(\cdot)=\min b r_{2}(\cdot)$ and is Country 2's least preferred Cournot-Nash equilibrium.

When countries' strategies are substitutable and $N=2$, a Cournot-Nash equilibrium in pure strategies always exists. The aim of Corollary 1 is to define an order on the preferences of each country. A direct consequence of the set of assumptions $A_{1}$ is that best responses are decreasing. In considering the opposite sign on the strategy set of Country 2, we can establish that the set of Cournot-Nash equilibria has a smallest and a highest element. The highest element is $(\bar{x}, \underline{y})$ and it is the highest equilibrium for Country 1, i.e. providing him the highest payoff of the simultaneous game. This equilibrium is also the least preferred by Country 2 . We can also define the point $(\underline{x}, \bar{y})$ as the smallest element in the set of Cournot-Nash equilibria and yielding to the highest payoff for Country 2 and the smallest payoff for Country 1.

With Proposition 2 and its corollary, we proceed as well to establish the conditions of existence of any Cournot-Nash equilibrium when countries' strategies are complementary. In this case the use of the theorems of the theory of supermodular games is direct.

\section{Proposition 2:}

Under the set of assumptions $A_{2}$, the global emission game is supermodular with the natural order on countries' strategy sets, and $C^{N}$ is non empty.

If individual emission levels ( $x$ and $y$ ) are increasing, the global emission level $(z)$ must also be increasing. Here the global consistency of the game is provided by the strict concavity of the benefit functions. Under the set of assumptions $\mathrm{A}_{2}$, we can provide the following corollary to Proposition 2.

\section{Corollary 2:}

Under the set of assumptions $A_{2}$, the extreme selections of the best response correspondence $b r_{i}(\cdot)$ are non decreasing in the rival's emissions. Hence, the smallest Cournot-Nash equilibrium $(\underline{x}, \underline{y})$ belongs to $C^{N}$ and both countries prefer it to all other Cournot-Nash equilibria.

Under $A_{2}$, the global emission game is naturally a supermodular game and best response correspondences are strictly increasing. The set of equilibria of the simultaneous game is thus non empty and has a smallest and a highest element Pareto ordered. In this case, the most preferred 
equilibrium for the countries (i.e. leading to the highest payoffs) is the one where both choose their lowest emission level $(\underline{x}, \underline{y})$. On the contrary, the equilibrium point $(\bar{x}, \bar{y})$ provides the lowest payoffs of the simultaneous game. This relation between emission levels and equilibrium payoffs is linked to the nature of the externalities - negative, generated by countries' strategic behaviours.

Let us now characterize the set of Stackelberg equilibria through Proposition 3. With respect to the works of Hellwig and Leininger (1987), this set is non empty independently of the sets of assumptions $\mathrm{A}_{1}$ and $\mathrm{A}_{2}{ }^{29}$

\section{Proposition 3:}

Under the set of assumptions $A_{1}$ [respectively $A_{2}$ ], the set of Stackelberg equilibrium strategies with country $i$ as leader is such that $S_{i}=\arg \max _{x i \geq 0}\left\{f_{i}(x, y):(x, y) \in G R \underline{b r}_{j}(\cdot)\right\}$. Hence, every point in $S_{i}$ gives the leader the same payoff, which is at least as high as his best Cournot-Nash payoff.

By Proposition 3, any Stackelberg equilibrium lies on the lowest best response correspondence of the follower. Moreover, even if the leader has several strategies maximizing its payoff, we can always assume the uniqueness (its payoff function being continuous in the strategy of the follower): if there is a multitude of equilibria for the leader, he can always choose the emission level yielding to the highest payoff for her. Furthermore given Corollaries 1 and 2, the most preferred Cournot-Nash equilibrium always lies on the lowest best response correspondence of the rival. As the leader can always choose its preferred equilibrium emission level which lies on the same correspondence, it follows that any strategy for the leader brings him a payoff at least as high as the one he receives at the equilibrium of the simultaneous game.

Given the equilibria of the simultaneous and of the sequential games, we now compare the associated payoffs to establish the SPE of the extended game.

\section{Results : Subgame perfect equilibria of the extended game and their respective environmental consequences}

This section determines the conditions under which both countries choose the same position in the global emission game and those under which the positions chosen differ. The choice for one alternative is directly linked to the nature of the interactions between the countries. So the following propositions strongly rely on the existence results of the previous section. In each case, we also discuss the environmental impact of countries' strategic behaviours with regard to the alternative not chosen. The next proposition provides the conditions under which the endogenous determination of the sequence of choices yields to the simultaneous game and thus to one Cournot-Nash equilibrium.

\footnotetext{
${ }^{29}$ The authors establish that, for compact strategy sets and continuous payoff functions, Stackelberg equilibria always exist and Stackelberg equilibrium strategy sets $\left(S_{i}, i=1,2\right)$ are non empty. These conditions are true under both sets of assumptions $\mathrm{A}_{1}$ and $\mathrm{A}_{2}$.
} 


\section{Proposition 4:}

Under the set of assumptions $A_{1}$, assume that no Cournot-Nash equilibrium lies on a boundary (i.e. with one emission level being 0). Then the set of subgame perfect Nash equilibria of the extended game is such that $E=\left\{(e, e), C^{N}\right\}$.

When countries' strategies are substitutable, the sole outcome of the extended game is the one in which the countries act simultaneously. The reason is twofold: both countries prefer their payoff as the leader and second, they will do all that is possible not to be the follower. The reason is that their payoff as the follower is always below the one they can get at any Cournot-Nash equilibrium. ${ }^{30}$

From an environmental impact point of view, this case is the most difficult to interpret. It appears through the proof of Proposition 4 that the leader would have higher emissions, whereas the follower would have lower emissions with regard to the solution of the simultaneous game. The conclusion on the environmental impact of this SPE thus depends on the strength of the substitutability between countries' strategies. Two cases are relevant: substitutability is weak or substitutability is strong. ${ }^{31}$ The solution of the simultaneous game is preferable from a global point of view (to the sequential one) when countries' strategies are weakly substitutable: the SPE of Proposition 4 leads to a lower global equilibrium emission level than if a leader were to emerge because in this case, an increase of emissions by the leader would not be compensated by the decrease of emissions by the follower.

This assertion becomes false when countries' strategies are strongly substitutable and the equilibrium is interior: in this case, an increase of emissions by the leader would now be completely compensated by the decrease of emissions by the follower. Therefore the SPE of Proposition 4 is not anymore the most favourable outcome from a global point of view when countries' strategies are strongly substitutable.

The following proposition provides the conditions under which the endogenous determination of the sequence of choices yields to one of the sequential modes of play: if a country chooses to play first in the first stage, the other will choose to play second and vice versa. These conditions lead to the emergence of a leader in the global emission game.

\section{Proposition 5:}

Under the set of assumptions $A_{2}$, the set of subgame perfect Nash equilibria of the extended game is such that $E=\left\{(e, l), S_{1}\right\} \cup\left\{(l, e), S_{2}\right\}$.

When countries' strategies are complementary, the solution of the extended game is such that one of the two leader-follower configurations emerges. The reason is that even if both countries prefer their payoff as the leader, their payoff at any Cournot-Nash equilibrium is always lower than the one a

\footnotetext{
${ }^{30}$ For strictly positive emission levels (the equilibrium is interior), we show in Appendix B that the sets of Cournot-Nash and Stackelberg equilibria are always distinct (See Lemma B.1).

${ }^{31}$ Each case depends on the assumption on the benefit functions (cf. the discussion following Proposition 1)
} 
country gets as the follower. Consequently if Country 1 anticipates that Country 2 will choose to act first, he will act second and vice versa. ${ }^{32}$

Another striking point is that the individual equilibrium emission levels of the sequential game are below those of the simultaneous game: both countries choose to reduce their emission level with regard to the Pareto dominant emission levels of the simultaneous game. Therefore the position of leadership does not necessarily mean that the leader will increase its emissions to the detriment of the follower. As the strategies are complementary, the feedback is positive and both countries reduce their individual emission level. We can thus conclude without any ambiguity that the SPE of the extended game underlined by Proposition 5 is the best outcome from an environmental point of view with regard to the simultaneous one.

The fact remains that a limit to Proposition 5 is that both leader-follower configurations can emerge. The proposition cannot endogenously explain why a country should be a leader or a follower. The following proposition provides sufficient conditions under which a specific leader emerges in the global emission game.

\section{Proposition 6:}

Consider for example that Country 1's payoff function checks the set of assumptions $A_{1}$ and that Country 2's payoff function checks the set of assumptions $A_{2}$. If an interior Cournot-Nash equilibrium exists, then the set of subgame perfect Nash equilibria of the extended game is such that $E=\left\{(e, l), S_{1}\right\}$.

When the nature of the interactions between the countries differs, Proposition 6 lets us conclude that the leader is the country whose payoff function presents strategic substitutability. Given Proposition 4, we know that such a country will do all he can not to be the follower. Therefore the strategy "Late" in the first stage of the extended game is strictly dominated for Country 1. On the contrary the country whose payoff function exhibits strategic complementarities always prefers to be a follower, instead of the outcome where both countries take action simultaneously (See Proposition 5). In this configuration, strategic behaviours of both countries are well matched and a specific leader emerges.

Note that in this case, we need to assume the existence of an equilibrium point in the simultaneous game. As best response correspondences are not necessarily continuous, it's possible that no CournotNash equilibrium exists; but if there exists one, it's necessarily unique. This point is linked to the fact that best response strategies progress in opposite directions (the one of Country 1 is strictly decreasing, whereas the one of Country 2 is strictly increasing). A sufficient condition to guarantee the existence of such an equilibrium point is that the payoff functions are quasi concave in their own strategy: this assumption ensures continuous best response strategies that necessarily intersect.

\footnotetext{
${ }^{32}$ For strictly positive emission levels, Lemma B.2 in Appendix B shows that the sets of Cournot-Nash and Stackelberg equilibria are always distinct.
} 
The last result is also interesting when considering the environmental impact of countries' strategies. This configuration is such that the leader as well as the follower reduce their emission level with regard to the simultaneous case, yielding a lower aggregated emission level and thus a better environment.

\section{Conclusion}

The paper confirms the widespread perception that the Stackelberg concept is unsuitable under the usual assumptions of the global emission game, i.e. when countries' strategies are substitutable. In this case, each country has an incentive to be the leader (and certainly not a follower), and both are led back to one of the Cournot-Nash equilibria of the simultaneous game. Thus considering the existence of negative leakages between countries' economic activities - as postulated in the existing literature on international cooperation to control GHG emissions, no country or group should emerge as a leader in the implementation of its environmental policy.

Nonetheless conditions yielding to sequential outcomes also exist: a leader can emerge endogenously. These cases are those in which the payoff function of one or both countries exhibits strategic complementarities. A striking point under these circumstances is that both the leader and the follower reduce their individual emissions with respect to the simultaneous alternative. From an economic point of view, it means that a country (or a group) can choose to implement a stronger national environmental policy than the others. In this prospect, he will be followed by the others: they will reduce as well their emissions even if not necessarily as much as the leader. A second mover advantage can also appear depending on the strength of the interactions between countries. Hence, if reinforcement effects exist between countries' economic activities (because of increasing return to scale, transfers of technologies, imitation), we should observe the emergence of a country (or a group) leading in the implementation of its environmental policy.

Finally, the set of propositions in this paper relies on minimal assumptions, including the absence of technical ones, such as concavity of payoffs and uniqueness of Cournot-Nash and Stackelberg equilibria. Hence, a sufficient condition for our analysis is the one of global monotony of the best responses. For all the cases studied, the interiority assumptions are needed only to ensure that both equilibrium concepts do not coincide. This allows us to provide clear predictions on countries' behaviours in the extended global emission game and ensures that sequential and simultaneous outcomes are not equilibria at the same time.

\section{Acknowledgements}

This paper has benefited from fruitful comments from participants at the $11^{\text {th }}$ annual conference of INFER (Stirling, 2009); at the Environment and Natural resources TSE group Workshop (LERNA, Toulouse, 2010); at the conference of ADRES (Lyon, 2010); and at the "Eco-Opti" Seminar (ParisNanterre, 2010). I am also particularly grateful to François Salanié, Dirk Ruebbelke, Claire Fitzgerald, 
Jérémy Celse and Anil Markandya for their precious advices and help throughout the writing of this paper.

\section{Appendix A: Definitions and main theorems of lattice theory}

Definitions and theorems introduce in this appendix are only a simplified version of the ones of lattice theory. We take the parameter and action sets, respectively $T$ and $X$, to be compact subsets of $I R$, and $X_{t}$ a correspondence from $T$ to $X$, with $X_{t}$ being the set of feasible actions when the parameter is $t$.

\section{Definition A.1:}

A function $f: X \times T \rightarrow I R$ has [strictly] increasing differences in $(x, t)$ if for all $x^{\prime} \geq x$ and $t^{\prime} \geq t$ : $f\left(x^{\prime}, t^{\prime}\right)-f\left(x, t^{\prime}\right) \geq[>] f\left(x^{\prime}, t\right)-f(x, t)$.

\section{Lemma A.1 (see Amir, 2003, p. 5 for the proof):}

If $f$ is twice continuously differentiable, $f$ has [strictly] increasing differences in $(x, t)$ if and only $\partial^{2} f(x, t) / \partial x \partial t \geq[>] 0$ for all $x$ and $t$.

For functions defined on $I R^{2}$, increasing differences is equivalent to supermodularity, so the two terms can be used interchangeably.

\section{Definition A.2:}

A function $f: X \rightarrow I R$ is upper semi-continuous in $x_{0}$ if $\limsup _{x \rightarrow x_{0}} f(x) \leq f\left(x_{0}\right)$. A function $f$ is upper semi-continuous if it is for all $x_{0} \in X$.

\section{Definition A.3:}

For $t \in R_{+}$, let $X_{t}=[g(t), h(t)] \subset R_{+}$, with $g(\cdot)$ and $h(\cdot)$ being real valued functions and with $g \leq h$. $X_{t}$ is ascending [descending] in $t$ if $g(\cdot)$ and $h(\cdot)$ are increasing [decreasing] in $t$.

A non cooperative game is a triple $\left(N, X_{i}, f_{i}\right)$ consisting of a non empty set of players $N$, a set $X_{i}$ of feasible individual strategies, and a payoff function $f_{i}$ defined on $\times_{i=1}^{i=n} X_{i}$ for each player $i$ in $N$.

\section{Definition A.4:}

A non-cooperative game $\left(N, X_{i}, f_{i}\right)$ is a supermodular game if each set $X_{i}$ of feasible strategies is a compact set of the Euclidian space and if each payoff function $f_{i}\left(x_{i}, x_{-i}\right)$ is upper semi-continuous in $x_{i}$ and has increasing differences in $\left(x_{i}, x_{j}\right)$ for all players $i, j \in N$ and $i \neq j$.

\section{Lemma A.2 (Topkis, 1998, chapter 4):}

The set of all equilibrium points for a non cooperative game $\left(N, X_{i}, f_{i}\right)$ is identical to the set of fixed points for the best joint response correspondence, i.e. the direct product of players' individual best response correspondences $\times_{i=1}^{i=n} b r_{i}(\cdot)$.

\section{Theorem A.1 (Topkis, 1978):}

If $f: X \times T \rightarrow I R$ is upper semi-continuous and has increasing [decreasing] differences in $(x, t)$, and $X_{t}$ is ascending [descending] in $t$, then the maximum and minimum selections of $x^{*}(t)=\operatorname{argmax}_{x \in X t} f(x, t)$ are increasing [decreasing] in $t$. If $f$ has strictly increasing [decreasing] differences in $(x, t)$, then the conclusion of theorem holds for every selection of $x *(\cdot)$. 


\section{Theorem A.2 (Topkis, 1979):}

If $f: X \times T \rightarrow I R$ is upper semi-continuous in $x$ for each $t$, and $f$ has increasing differences in $(x, t)$ then: $(i) x(t)=\operatorname{argmax}_{x \in X} f(x, t)$ is non empty and possess maximal and minimal selections, $\bar{x}(t)$ and $\underline{x}(t)$ for all $t$; (ii) maximal and minimal selections of $x(t)$ are increasing functions of $t$. Furthermore, if $f$ has strictly increasing differences in $(x, t)$, then every selection of $x(t)$ is increasing.

\section{Theorem A.3 (Tarsky, 1955):}

Let $X$ be an non empty and compact interval of the Euclidian space and let $f: X \rightarrow X$ be an increasing function $(f(x) \leq f(y)$ if $x \leq y)$. Then the set of fixed points of $f$ is non empty and contains a smallest and a largest element in $X$.

\section{Theorem A.4 (Milgrom and Roberts, 1990):}

Let $\underline{x} *$ and $\bar{x} *$ denote the smallest and largest elements of $X$, and suppose $y$ and $z$ are two equilibria with $y \geq z$. (1) If $f_{i}\left(\underline{x}_{i}, x_{-i}\right)$ is increasing in $x_{-i}$, then $f_{i}(y) \geq f_{i}(z)$. (2) If $f_{i}\left(\underline{x}_{i}, x_{-i}\right)$ is decreasing in $x_{-i}$, then $f_{i}(y) \leq f_{i}(z)$. If the condition in (1) holds for some subset of players $N_{1}$ and the condition in (2) holds for the remainder $M N_{l}$, then the largest equilibrium is the most preferred equilibrium for the players in $N_{l}$, and the least preferred for the remaining players. The smallest equilibrium is the least preferred by the players in $N_{1}$, and the most preferred by the remaining players.

\section{Appendix B: Proofs}

This appendix contains the proofs of propositions made in the text. We also introduce intermediate results (Lemma B.1 and B.2) that are useful building blocks to simplify proofs of Propositions 4, 5 and 6. Propositions 1 and 2 and their respective corollaries (Corollary 1 and 2) provide the conditions under which the two country game possesses at least one pure strategy Cournot-Nash equilibrium $\left(C^{N}\right.$ non empty) and characterize properties of equilibria given the nature of interactions between countries. Proposition 3 does the same for Stackelberg equilibria $\left(S_{i}\right.$ non empty, $\left.i=1,2\right)$. Finally, by comparing equilibrium payoffs, we can conclude on the subgame perfect equilibria (SPE) of the extended game (Propositions 4, 5 and 6).

Reminder:

- The functions $B: I R_{+} \rightarrow I R_{+}$and $D: I R_{+} \rightarrow I R_{+}$are twice continuously differentiable and non decreasing. The analysis is split into two distinct sets of assumptions:

○ Under $\mathrm{A}_{1}$, the damage function $D_{i}(\cdot),(i=1,2)$ is strictly convex, $\forall x, y \geq 0$ and $\exists \bar{Z}_{i} \in\left[0, K_{i}\right]$ such that $B_{i}(Z)-D_{i}(Z) \leq B_{i}\left(\bar{Z}_{i}\right)-D_{i}\left(\bar{Z}_{i}\right), \forall Z(i=1,2)$ : countries' strategies are substitutable;

○ Under $\mathrm{A}_{2}$, both the benefit function $B_{i}(\cdot),(i=1,2)$ and the damage function $D_{i}(\cdot),(i=1,2)$ are strictly concave: countries' strategies are complement.

- $C^{N}, S_{i}$ and E denote respectively the set of Cournot-Nash equilibrium strategies, the set of Stackelberg equilibrium strategies with player $i$ as leader and the set of SPE of the extended game (in no case, uniqueness is required).

\section{Proof of Proposition 0:}

The proof of Proposition 0 comes directly from the strategic form of the extended game (see Figure

2). Countries' best responses are such that, if Country 1 anticipates that:

- Country 2 play "Early", it chooses to play "Early" if $f_{l}^{C N}>f_{l}^{F}$ and "Late" if not;

- Country 2 play "Late", it always chooses to play "Early" as $f_{l}^{L}>f_{l}^{C N}$ by assumption.

Idem for Country 2, if it anticipates that:

- Country 1 play "Early", it chooses to play "Early" if $f_{2}^{C N}>f_{2}{ }^{F}$ and "Late" if not;

- Country 1 play "Late", it always chooses to play "Early" as $f_{2}^{L}>f_{2}^{C N}$ by assumption.

Combining countries' best responses leads to the conclusions of Proposition 0. 


\section{Proof of Proposition 1:}

Strategy sets are compact subsets of the real and such that $X_{i}=\left[0, K_{i}\right], i=1,2$. As benefit and damage functions are continuous, both countries' payoff functions are continuous in their own strategy. Then if damage functions are strictly convex, payoff functions have strictly decreasing differences in $(x, y)$ :

and:

$$
\begin{aligned}
& f_{i}(x, y)=B_{i}(x)-D_{i}(x+y), \quad \forall i=1,2, \\
& \frac{\partial^{2} f_{i}(x, y)}{\partial x \partial y}=-D_{i}^{\prime \prime}(x+y)<0, \quad \forall i=1,2 .
\end{aligned}
$$

Under these conditions, any selection of a country's best response correspondence is non increasing in the strategy of the other country: for Country $1, b r_{1}(y)=\arg \max _{x \in X_{1}} f_{1}(x, y)$ is non increasing in $y$, while for Country 2, $b r_{2}(x)=\arg \max _{y \in X_{2}} f_{2}(x, y)$ is non increasing in $x$ (see Theorem A.1, Topkis, 1978, Appendix A).

Secondly, the actual strategy set of a country is $\left[0, \bar{Z}_{i}\right]$, with $\bar{Z}_{i}$ being the emission level a country adopts when it's sole to pollute (i.e. $\bar{Z}_{i}=b r_{i}(0)$ ). When best responses are monotone decreasing, $\bar{Z}_{i}$ is also the highest equilibrium emission level a country can choose.

Reversing the natural order on one country's emission set, for example Country 2, payoff functions then present increasing differences in $(x,-y)$. Under the set of assumptions $\mathrm{A}_{1}$, the two-country global emission game is a supermodular game. By Theorems A.2 (Topkis, 1979) and A.3 (Tarski, 1955) in Appendix A, the set $C^{N}$ is non empty and has a smallest and a largest element.

\section{Proof of Corollary 1:}

Under $\mathrm{A}_{1}, C^{N}$ is non empty and extreme selections of the best response correspondence of a country are decreasing in the emission level of the rival (with the natural order on the strategy sets) by Theorem A.1 (Appendix A). Reversing the natural order on Country 2's strategy set, extreme selections become increasing, respectively in $-y$ for Country 1 and in $x$ for Country 2 . Consequently the maximal selection in $C^{N}$ is $(\bar{x},-\bar{y})$ in the new order or equivalently, $(\bar{x}, \underline{y})$ in the natural order.

The proof that $(\bar{x}, \underline{y})$ lies on $\underline{b r}_{2}(\cdot)$ is given by contradiction: suppose that $(\bar{x}, \underline{y}) \notin G r \underline{b r_{2}}(\cdot)$ and that there is a fixed point $\left(x^{\prime}, y^{\prime}\right) \in C^{N}$ with $\left(x^{\prime}, y^{\prime}\right) \in G r \underline{b r}_{2}(\cdot)$. It means that $y^{\prime}<\underline{y}$ or equivalently $b r_{2}\left(x^{\prime}\right)<b r_{2}(\bar{x})$. As with the natural order on the strategy sets, $\underline{b r_{2}}(\cdot)$ is decreasing we find that $x^{\prime}>\bar{x}$. Both $x^{\prime}>\bar{x}$ and $y^{\prime}<\underline{y}$ contradicts the extreme nature of $(\bar{x}, \underline{y})$.

Finally the fact that $(\bar{x}, \underline{y})$ is the least preferred by Country 2 is a direct consequence of Theorem A.4 (Milgrom and Roberts, 1990).

\section{Proof of Proposition 2:}

Under $\mathrm{A}_{2}$, the global emission game is naturally a supermodular game, i.e. countries' payoff functions have strictly increasing differences in $(x, y)$. By Theorem A.2 (Topkis, 1979), best responses are thus strictly increasing and by Theorem A.3 (Tarski, 1955), $C^{N}$ is non empty.

\section{Proof of Corollary 2:}

Under $\mathrm{A}_{2}$, both countries' payoff functions have strictly increasing differences in $(x, y)$ and by applying Theorem A.2 (Topkis, 1979), any selection of a country best response is increasing in the strategy of the other country. For Country $1, x *(y)=\arg \max _{x \in X_{1}} f_{1}(x, y)$ is increasing in $y$, whereas for Country $2, y^{*}(x)=\arg \max _{y \in X_{2}} f_{2}(x, y)$ is increasing in $x$. As the global emission game is a game of negative externality, Theorem A.4 (Milgrom and Roberts, 1990) lets us conclude that the smallest element in $C^{N}$, i.e. $(\underline{x}, \underline{y})$, is also the Pareto dominant equilibrium; the highest element in $C^{N}$, i.e. $(\bar{x}, \bar{y})$, is the Pareto dominated equilibrium. The former provides the highest possible equilibrium payoff for both countries and the latter the worst equilibrium payoff for both of them. The proof is 
trivial: let $\left(x^{*}, y^{*}\right)$ and $\left(x^{\prime}, y^{\prime}\right)$ be two Nash equilibria in pure strategies such that $\left(x^{*}, y^{*}\right)>\left(x^{\prime}, y^{\prime}\right)$. Then $f\left(x^{\prime}, y^{\prime}\right) \geq f\left(x^{*}, y^{\prime}\right) \geq f\left(x^{*}, y^{*}\right)$. The first inequality is due to the fact that $\left(x^{\prime}, y^{\prime}\right)$ is an equilibrium and the second inequality is because $f(\cdot)$ is decreasing in $y$. Equilibria of the game are thus ordered, the smallest being Pareto dominant.

\section{Proof of Proposition 3:}

Proposition 3 aims at characterizing the set of Stackelberg equilibria in the global emission game whatever the nature of interactions between countries. The proof relies on the assumptions on the benefit and damage functions, the latter defining the properties of the follower's best response correspondence on which lies the Stackelberg equilibrium. We thus consider respectively each set of assumptions $A_{1}$ and $A_{2}$. Under $A_{1}$ or $A_{2}$, continuity of payoff functions in countries' own strategy insures that the follower's best response correspondence has a closed graph. In what follows, we assume that Country 1 acts as a leader and Country 2 as a follower. Consequently Country 2's best response correspondence has a lowest element $\underline{b r}_{2}(\cdot)$ and we aim at showing that any Stackelberg equilibrium lies on this minimal reaction curve.

i) Under $\mathrm{A}_{1}$, the minimal reaction curve $\underline{b r}_{2}(\cdot)$ is at least lower semi-continuous and rightcontinuous. Yet, a strictly decreasing reaction curve, if discontinuous, can only jump down. By contradiction, suppose that there is a Stackelberg equilibrium $\left(x^{S}, y^{S}\right)$ such that $\left(x^{S}, y^{S}\right) \notin G R \underline{b r}_{2}(\cdot)$ and $y^{S}>\underline{b r}_{2}\left(x^{S}\right)$. By Corollary 1 , any selection of $b r_{2}(\cdot)$ is non increasing with the natural order on the strategy sets. Hence the (finite) set of points at which $b r_{2}(\cdot)$ is not uniquely defined coincides with the one at which $\underline{b r}_{2}(\cdot)$ is discontinuous. By the contradiction assumption, $b r_{2}(\cdot)$ is multi-valued at the point $x^{S}$. We can thus find a sufficiently small $\varepsilon>0$ such that choosing $x^{S}+\varepsilon$ for the leader would lead to a unique best reply by the follower that is strictly smaller than $y^{S}$. It means that $b r_{2}(\cdot)$ is single valued at $x^{S}+\varepsilon$, with $y^{S}>\underline{b r}_{2}\left(x^{S}\right)$ as $\underline{b r_{2}}(\cdot)$ is right-continuous. The payoff of the leader is then such that $f_{1}\left(x^{S}+\varepsilon, \underline{b r}_{2}\left(x^{S}+\varepsilon\right)\right)>f_{1}\left(x^{S}, y^{S}\right)$ as $f_{1}(x, y)$ is continuous in $x$ and strictly decreasing in $y$. But this assertion contradicts the initial statement that $\left(x^{S}, y^{S}\right)$ is a Stackelberg equilibrium. It follows that $\left(x^{S}, y^{S}\right) \in G R \underline{b r}_{2}(\cdot)$ and that $S_{1}=\operatorname{argmax}_{x \geq 0} f_{1}\left(x, \underline{b r_{2}}(x)\right)$.

What about the existence of multiple Stackelberg equilibria. If they exist, it's always relative to $\underline{b r}_{2}(\cdot)$ which is a parameter in the payoff function of the leader. Yet, if the leader has to choose between several equilibria, he will always adopt the strategy leading to the highest payoff. Therefore we can assume the uniqueness of the Stackelberg equilibrium, with any point in $S_{l}$ leading to the same payoff for the leader.

Finally, as Country 1's most preferred Cournot-Nash equilibrium is $(\bar{x}, \underline{y})$ with $(\bar{x}, \underline{y}) \in G R \underline{b r}_{2}(\cdot)$, the payoff of the leader is at least as high as the one he would obtain at his most preferred equilibrium of the simultaneous game.

ii) Under $\mathrm{A}_{2}$, the minimal reaction curve $\underline{b r}_{2}(\cdot)$ is at least upper semi-continuous and leftcontinuous. Yet, a strictly increasing reaction curve, if discontinuous, can only jump up. We adopt the same reasoning as before, except that we consider $x^{S}-\varepsilon$ with $\varepsilon>0$, instead of $x^{S}+\varepsilon$. This deviation is possible only if $x^{s} \neq 0$. Now, by contradiction, suppose that there is a Stackelberg equilibrium $\left(x^{S}, y^{S}\right)$ such that $\left(x^{S}, y^{S}\right) \notin G R \underline{b r}_{2}(\cdot)$ and $y^{S}>\underline{b r}_{2}\left(x^{S}\right)$. By Corollary 2, any selection of $b r_{2}(\cdot)$ is non decreasing with the natural order on the strategy sets. Hence the (finite) set of points at which $b r_{2}(\cdot)$ is not uniquely defined coincides with the one at which $\underline{b r}_{2}(\cdot)$ is discontinuous. By the contradiction assumption, $b r_{2}(\cdot)$ is multi-valued at the point $x^{S}$. We can thus find a sufficiently small $\varepsilon>0$ such that choosing $x^{S}-\varepsilon$ for the leader would lead to a unique best reply by the follower that is strictly smaller than $y^{S}$. It means that $b r_{2}(\cdot)$ is single valued at $x^{S}-\varepsilon$, with $y^{S}>\underline{b r}_{2}\left(x^{S}\right)$ as $\underline{b r}_{2}(\cdot)$ is left-continuous. 
The payoff of the leader is then such that $f_{1}\left(x^{S}-\varepsilon, \underline{b} \underline{r}_{2}\left(x^{S}-\varepsilon\right)\right)>f_{1}\left(x^{S}, y^{S}\right)$ as $f_{1}(x, y)$ is continuous in $x$ and strictly decreasing in $y$. But this assertion contradicts the initial statement that $\left(x^{S}, y^{S}\right)$ is a Stackelberg equilibrium. It follows that $\left(x^{S}, y^{S}\right) \in G R \underline{b r}_{2}(\cdot)$ and that $S_{1}=\operatorname{argmax}_{x \geq 0} f_{1}\left(x, \underline{b r}_{2}(x)\right)$.

As for point i) above, we can assume the uniqueness of the Stackelberg equilibrium and any point in $S_{1}$ leads to the same payoff for the leader. This payoff is at least as high as the one the leader would obtain at his most preferred equilibrium of the simultaneous game.

\section{Lemma B.1:}

Under the set of assumptions $A_{1}$, if all Cournot-Nash equilibria are interior (i.e. with no emission level being 0$)$, then the extreme Cournot-Nash equilibrium $(\bar{x}, \underline{y})$ is such that $(\bar{x}, \underline{y}) \notin S_{1}$.

\section{Proof:}

Under $\mathrm{A}_{1}$, a country's payoff function has strictly decreasing differences in $(x, y)$. Thus $\partial f(\cdot) / \partial x$ is strictly decreasing in $y$ and each country's best reply strategies are strictly decreasing. The proof relies on the interiority of the equilibrium. By contradiction, suppose that the argument maximum is constant, i.e. $y^{\prime}>y, x *\left(y^{\prime}\right)=x *(y)$. For any $y$, first order conditions are such that:

$$
\frac{\partial f(x, y)}{\partial x}=0 \quad \text { and } \quad \frac{\partial f\left(x, y^{\prime}\right)}{\partial x}=0 .
$$

Both equalities contradict the statement that $\partial f(\cdot) / \partial x$ is strictly decreasing in $y$. Hence any selection of $\operatorname{br}(\cdot)$ is strictly decreasing.

Now we show that Cournot-Nash and Stackelberg equilibria never coincide, i.e. that $(\bar{x}, y) \notin S_{1}$. $\operatorname{Suppose}\left(x^{S}, \underline{b r}_{2}\left(x^{S}\right)\right)$ any Stackelberg equilibrium with Country 1 as leader. As $(\bar{x}, \underline{y})$ is interior, it is such that $\partial f(\bar{x}, y) / \partial x=0$. If $\left(x^{S}, \underline{b r_{2}}\left(x^{S}\right)\right)$ is interior as well, then we can check the following first order condition:

$$
\frac{\partial f_{1}\left(x^{S}, \underline{b r}_{2}\left(x^{S}\right)\right)}{\partial x}+\frac{\partial f_{1}\left(x^{S}, \underline{b r}_{2}\left(x^{S}\right)\right)}{\partial y} \cdot{\underline{b r^{\prime}}}_{2}\left(x^{S}\right)=0 .
$$

As ${\underline{b r^{\prime}}}_{2}(\cdot)<0$ and $\partial f_{1} / \partial y<0$, we conclude that $\frac{\partial f_{1}\left(x^{S}, \underline{b r}_{2}\left(x^{S}\right)\right)}{\partial x}<0$. Hence $\bar{x} \neq x^{S}$ and $\underline{b r}_{2}(\bar{x}) \neq \underline{b r}_{2}\left(x^{S}\right)$. If the Stackelberg equilibrium is not interior, the same conclusion follows as $(\bar{x}, \underline{y})$ is necessarily interior.

\section{Proof of Proposition 4:}

Given Propositions 1 and 3, $C^{N}, S_{I}$ and $S_{2}$ are non empty. In more by Proposition 3 Country $i$ is better off at any point of $S_{i}$ than at any point of $C^{N}$. Finally, by Corollary $1,(\bar{x}, \underline{y}) \in C^{N}$ where $\underline{y}$ is Country 2 's lowest equilibrium emission level and also its worst equilibrium. By Proposition 0, we have still to show that a country prefers its worst Cournot-Nash equilibrium to its payoff as a follower. Suppose $\left(x^{S}, y^{s}\right)$ is the Stackelberg equilibrium with Country 1 as leader and $(\bar{x}, \underline{y})$ is its most preferred equilibrium when both choose their strategy simultaneously. By Lemma B.1, $\left(x^{S}, y^{S}\right) \neq(\bar{x}, \underline{y})$ even if both lie on $\underline{b r}_{2}(\cdot)$. By Proposition 3, we know that Country 1 prefers its payoff as a leader to its most preferred Cournot-Nash payoff, i.e.:

$$
f_{1}\left(x^{s}, y^{s}\right)>f_{1}(\bar{x}, \underline{y}) .
$$

By definition of Nash equilibrium, we can also write the following inequality:

$$
f_{1}\left(x^{S}, y^{S}\right) \geq f_{1}\left(x^{S}, \underline{y}\right) \text {. }
$$


It means that if Country 2 chooses it Cournot-Nash equilibrium strategy, $x^{S}$ is not a best reply for Country 1 . As the game is of negative externality $\left(\partial f_{1}(x, y) / \partial y<0\right)$, the inequality (1) lets us conclude that $y^{S}<\underline{y}$, or equivalently $\underline{b r}_{2}\left(x^{S}\right)<\underline{b r}_{2}(\bar{x})$. As $\underline{b r}_{2}(\cdot)$ is strictly decreasing, it follows that $x^{S}>\bar{x}$.

For the follower, given the order defined on countries' individual emission levels at the CournotNash and Stackelberg equilibria, we find the following inequalities on its equilibrium payoffs:

$$
f_{2}\left(x^{s}, y^{s}\right)<f_{2}\left(\bar{x}, y^{s}\right)<f_{2}(\bar{x}, \underline{y}) .
$$

The first inequality in (2) is due to the fact that $x^{S}>\bar{x}$ and that Country 2's payoff is decreasing in the strategy of the other country (the game is of negative externality and $\left.\partial f_{2}(x, y) / \partial x<0\right)$. The second inequality is linked to the definition of Nash equilibrium: the payoff of Country 2 is maximum when the latter adopts its best response to $\bar{x}$. We can conclude that the follower always prefers its worst Cournot-Nash payoff of the simultaneous game rather than its payoff at the Stackelberg equilibrium. The proof when Country 2 is leader is the same and relies on the equilibrium $(\underline{x}, \bar{y})$. By Proposition 0 , the conditions studied here are the one under which the set of equilibria of the extended global emission game is $E=\left\{(e, e), C^{N}\right\}$.

\section{Lemma B.2:}

Under the set of assumptions $A_{2}$, if all Cournot-Nash equilibria are interior, then they never coincide with the Stackelberg equilibria; in particular $(\underline{x}, \underline{y}) \notin S_{1}$.

Proof:

The proof of Lemma B.2 is identical to the one of Lemma B.1 except that, under $\mathrm{A}_{2}$, a country's payoff function has strictly increasing differences in $(x, y)$. Thus $\partial f(\cdot) / \partial x$ is strictly increasing in $y$ and each country's best reply strategies are strictly increasing. As previously, the proof relies on the interiority of the equilibrium. By contradiction, suppose that the argument maximum is constant, i.e. $y^{\prime}>y, x *\left(y^{\prime}\right)=x *(y)$. For any $y$, first order conditions are such that:

$$
\frac{\partial f(x, y)}{\partial x}=0 \quad \text { and } \quad \frac{\partial f\left(x, y^{\prime}\right)}{\partial x}=0 .
$$

Both equalities contradict the statement that $\partial f(\cdot) / \partial x$ is strictly increasing in $y$. Hence any selection of $\operatorname{br}(\cdot)$ is strictly increasing. The proof that Cournot-Nash and Stackelberg equilibria never coincide is the same as for Lemma B.1.

\section{Proof of Proposition 5:}

Under $\mathrm{A}_{2}$, the global emission game is supermodular with the natural order on the strategy sets; countries' best responses are non decreasing. By Theorem A.4 (Milgrom and Roberts, 1990), the Pareto dominant equilibrium is the one with the lowest emission levels for both countries: $(\underline{x}, \underline{y}) \in C^{N}$. Moreover we know that a country $i$ is better off at any point of $S_{i}$ (i.e. as a leader) than at any point of $C^{N}$ (Proposition 3). By Proposition 0, we have to establish that a country always prefers its payoff as a follower to any other payoff resulting from the simultaneous game. To do so, consider $\left(x^{S}, y^{S}\right)$ as any Stackelberg equilibrium with Country 1 as leader. By Lemma B.2, we know that $\left(x^{S}, y^{S}\right) \notin C^{N}$. Moreover, by Proposition 3, Country 1 always prefers its payoff as a leader to any other payoff of the simultaneous game, and in particular to its most preferred Cournot-Nash payoff i.e.:

$$
f_{1}\left(x^{S}, y^{S}\right)>f_{1}(\underline{x}, \underline{y}) \text {. }
$$

Another inequality follows from the definition of Nash equilibrium: if Country 2 chooses its equilibrium strategy of the simultaneous game, $x^{S}$ is not a best response for Country 1 . Then:

$$
f_{1}\left(x^{S}, y^{S}\right) \geq f_{1}\left(x^{S}, \underline{y}\right) \text {. }
$$


As the game is of negative externality $\left(\partial f_{1}(x, y) / \partial y<0\right)$, inequality (3) lets us conclude that $y^{S}<\underline{y}$, or equivalently $\underline{b r}_{2}\left(x^{S}\right)<\underline{b}_{2}(\underline{x})$. As $\underline{b r}_{2}(\cdot)$ is strictly increasing by Lemma B.2, it follows that $x^{S}<\underline{x}$.

For the follower, given the order defined on countries' individual emission levels at the CournotNash and Stackelberg equilibria, we find the following inequalities on its equilibrium payoffs:

$$
f_{2}(\underline{x}, \underline{y})<f_{2}\left(x^{S}, \underline{y}\right)<f_{2}\left(x^{S}, y^{S}\right) .
$$

The first inequality of (4) is due to the fact that $x^{S}<\underline{x}$ and that Country 2's payoff is decreasing in the strategy of the other country (the game is of negative externality and $\left.\partial f_{2}(x, y) / \partial x<0\right)$. The second inequality is linked to the definition of Nash equilibrium: Country 2's payoff is maximum when it adopts its best response to $x^{S}$. We conclude that the leader and the follower always prefer their respective payoff at the Stackelberg equilibrium than their most preferred Cournot-Nash payoff of the simultaneous game. By Proposition 0, the conditions studied here are the one under which the set of equilibria of the extended global emission game is $E=\left\{(p, s), S_{1}\right\} \cup\left\{(s, p), S_{2}\right\}$.

\section{Proof of Proposition 6:}

Proposition 0 leads to a particular sequence of moves in the extended global emission game if: i) a country $i$ is better off at any point of $S_{i}$ than at any point of $C^{N}$; ii) Country 1 is better off at its least preferred equilibrium in $C^{N}$ than at any point in $S_{2}$, whereas Country 2 is better off at any point in $S_{2}$ than at any point in $C^{N}$. Whatever the set of assumptions $A_{1}$ or $A_{2}$, point i) is true by Lemma B.1. Preferences in point ii) can only be established if $C^{N}$ is non empty: to use Lemma B.1 and B.2, we have to assume the existence of an interior equilibrium in the simultaneous move game.

Under $A_{1}$, Country 1's payoff function presents strictly decreasing differences and by Lemma B.1, any selection of $b r_{1}(\cdot)$ is thus strictly decreasing. Similarly, under $\mathrm{A}_{2}$, Country 2's payoff function presents strictly increasing differences and by Lemma B.2, any selection of $b r_{2}(\cdot)$ is thus strictly increasing. Given the monotony of best responses for both countries and their direction, if an equilibrium exists, it is necessarily unique. Call it $\left(x^{*}, y^{*}\right)$. Then the proof of Proposition 6 is identical to the ones of Propositions 4 and 5. Note $\left(x^{1}, y^{1}\right)$ the Stackelberg equilibrium with Country 1 as leader. By Proposition 3 and the definition of Nash equilibrium, we can write the two following inequalities:

$$
f_{1}\left(x^{1}, y^{1}\right)>f_{1}\left(x^{*}, y^{*}\right) \quad \text { and } \quad f_{1}\left(x^{1}, y^{1}\right) \geq f_{1}\left(x^{1}, y^{*}\right) .
$$

The game being of negative externality, we deduce that $y^{1}=b r_{2}\left(x^{1}\right)<b r_{2}\left(x^{*}\right)=y^{*}$. As $b r_{2}(\cdot)$ is strictly increasing, it follows that $x^{1}<x *$. Finally for the follower, the inequalities are such that (the reasoning is the same as for relation (4)):

$$
f_{2}\left(x^{*}, y^{*}\right)<f_{2}\left(x^{1}, y^{*}\right)<f_{2}\left(x^{1}, y^{1}\right) \text {. }
$$

It means that Country 2 prefers its payoff as a follower at the Stackelberg equilibrium to its payoff at the Cournot-Nash equilibrium.

To show that Country 1 prefers its Cournot-Nash payoff to its Stackelberg payoff as follower, we proceed as for the proof of Proposition 4. Suppose $\left(x^{2}, y^{2}\right)$ the Stackelberg equilibrium with Country 2 as leader, such that the two following inequalities are true:

$$
f_{2}\left(x^{2}, y^{2}\right)>f_{2}\left(x^{*}, y^{*}\right) \quad \text { and } \quad f_{2}\left(x^{2}, y^{2}\right) \geq f_{2}\left(x^{2}, y^{*}\right) .
$$

As countries' payoffs are decreasing in the strategy of the other (game of negative externality), $y^{2}=b r_{1}\left(x^{2}\right)<b r_{1}\left(x^{*}\right)=y^{*}$. As $b r_{1}(\cdot)$ is strictly decreasing, we deduce that $x^{2}>x^{*}$. It follows for the follower that (the reasoning is the same as for relation (2)):

$$
f_{1}\left(x^{2}, y^{2}\right)<f_{1}\left(x^{*}, y^{2}\right)<f_{1}\left(x^{*}, y^{*}\right) .
$$

Meaning that Country 1 always prefers its payoff at the Cournot-Nash equilibrium to the one it obtains at the Stackelberg equilibrium as follower.

To conclude, under the assumptions of Proposition 6, the unique SPE of the extended game is such that Country 1 (with strategic substitutability) plays as a leader, whereas Country 2 (with strategic complementarities) plays as a follower. 


\section{References}

Amir, R., 2005. Supermodularity and complementarity in economics: an elementary survey. Southern Economic Journal, 71, 636-660.

Amir, R., Grilo, I., 1999. Stackelberg versus Cournot equilibrium. Games and Economic Behavior, 26, 1-21.

Barrett, S., 1994. Self-enforcing international environmental agreements. Oxford Economic Papers, 46, 878-894.

Barrett, S., 2003. Environment and Statecraft: The strategy of Environmental Treaty-Making. Oxford University Press, Oxford.

Barrett, S., 2005. The Theory of International Environmental Agreements. In: Handbook of Environmental Economics. Economywide and International Environmental Issues. North-Holland, Amsterdam and San Diego, 1457-1516.

Boadway, R., Song, Z., Tremblay, J-F., 2007. Commitment and matching contributions to public goods. Journal of Public Economics, 91, 1664-1683.

Carraro, C., Siniscalco, D., 1993. Strategy for the international protection of the environment. Journal of public Economics, 52, 309-328.

Cooper, R., 1999. Coordination Games: Complementarities and Macroeconomics. Cambridge University Press.

Copeland, B., Taylor, S., 2005. Free trade and global warming: a trade theory view of the Kyoto Protocol. Journal of Environmental Economics and Management, 49, 205-234.

Diamantoudi, E., Sartzetakis, E., 2006. Stable international environmental agreements: an analytical approach. Journal of Public Economic Theory, 8, 247-263.

Dumas, P., Ha-Duong, M., 2005. An abrupt stochastic damage function to analyse climate policy benefits. In: Haurie, A. and Viguier, L. (eds), The Coupling of Climate and Economic Dynamics: Essays on Integrated Assessment. Springer, Amsterdam.

Finus, M., 2001. Game Theory and International Environmental Cooperation. New horizons in environmental economics. Elgar, Cheltenham.

Hamilton, J., Slutsky, S., 1990. Endogenous timing in duopoly games: Stackelberg or Cournot equilibria. Games and Economic Behavior, 2, 29-46.

Hellwig, M., Leininger, W., 1987. On the existence of subgame perfect equilibrium in infinite-action games of perfect information. Journal of Economic Theory, 43, 55-75.

Heugues, M., 2009a. International Environmental Cooperation: A New Eye on the Greenhouse Gas Emissions' Control. Working paper LAMETA 2009-04.

Heugues, M., 2009b. Une nouvelle mise en perspective des problèmes environnementaux globaux dans le cas du changement climatique: les impacts de la complémentarité stratégique entre pays. Thèse de doctorat, Université Montpellier I.

Hoel, M., 1991. Global environmental problems: the effects of unilateral actions taken by one country. Journal of Environmental Economics and Management, 20, 55-70.

Milgrom, P., Roberts J., 1990. Rationalizability, learning and equilibrium in games with strategic complementarities. Econometrica, 58, 1255-77.

Ortiz, R. A., Markandya, A., 2009. Integrated impact assessment models of climate change with an emphasis on damage functions: A literature review. BC3 Working Paper Series 2009-06. 
Pereau, JC., Rotillon, G., Tazdaït, T., 2002. Une taxonomie des différents comportements coopératifs possibles face aux problèmes environnementaux globaux: de la coordination à l'engagement unilateral. In: Rotillon, G. (éd.), Régulation environnementale: Jeux, Coalitions et Contrats. Economica, Paris.

Tarski, A., 1955. A lattice-theoretic fixed point theorem and its application. Pacific Journal of Mathematics, 5, 285-309.

Topkis, D., 1978. Minimizing a submodular function on a lattice. Operations Research, 26, 305-21.

Topkis, D., 1979. Equilibrium points in nonzero-sum n-person submodular games. Journal on Control and Optimization, 17, 773-787.

Topkis, D., 1998. Supermodularity and Complementarity. Princeton University Press, New Jersey.

Ulph, A., 2001. Environmental Policy, International Agreements, and International Trade. Oxford University Press, Oxford.

Vives, X., 1999. Oligopoly Pricing: Old Ideas and New Tools. MIT Press, Cambridge. 


\title{
BC3 WORKING PAPER SERIES
}

\author{
Basque Centre for Climate Change (BC3), Bilbao, Spain
}

The BC3 Working Paper Series is available on the internet at the following addresses:

\section{http://www.bc3research.org/lits_publications.html}

http://ideas.repec.org/s/bcc/wpaper.html

BC3 Working Papers available:

2010-07

2010-08

2010-09

$2010-10$

2010-11

2010-12

$2010-13$

2010-14

$2010-15$

2010-16

2010-17

2011-01

2011-02

Luis M. Abadie, Mikel González-Eguino and José M. Chamorro: Optimal Abandonment of Coal-Fired Stations in the EU

Dirk Rübbelke and Stefan Vögele: Impacts of Climate Change on European Critical Infrastructures: The Case of the Power Sector

Roger Fouquet: The Sustainability of 'Sustainable' Energy Use: Historical Evidence on the Relationship between Economic Growth and Renewable Energy

Karen Pittel and Dirk Rübbelke: Energy Supply and the Sustainability of Endogenous Growth

Ramon Arigoni Ortiz, Alexander Golub, Oleg Lugovoy, Anil Markandya and James Wang: The DICER Model: Methodological Issues and Initial Results.

Elena Ojea, Julia Martin-Ortega and Aline Chiabai: Classifying Ecosystem Services for Economic Valuation: the Case of Forest Water Services

Aline Chiabai, Chiara Travisi, Anil Markandya, Helen Ding, and Paulo Nunes: Economic Assessment of Forest Ecosystem Services Losses: Cost of Policy Inaction

Kaysara Khatun: Competing Ecosystem services: an Assessment of Carbon and Timber in the Tropical forests of Central America

Karen Pittel and Dirk Rübbelke: Local and Global Externalities, Environmental Policies and Growth

Margherita Grasso, Matteo Manera, Aline Chiabai, and Anil Markandya: The Health Effects of Climate Change: A Survey of Recent Quantitative Research

Luis Mari Abadie, Ramon Arigoni Ortiz and Ibon Galarraga: The Determinants of Energy Efficiency Investments in the U.S.

Roger Fouquet: Long Run Dynamics of Energy-Related External Costs

Dirk Rübbelke: International Support of Climate Change Policies in Developing Countries: Strategic, Moral and Fairness Aspects

Melanie Heugues: Endogenous Timing in Pollution Control: Stackelberg versus Cournot-Nash Equilibria 